\section{Extended Mie Theory for a Gyrotropic-Coated Conducting Sphere: An Analytical Approach}

\author{
You-Lin Geng and Cheng-Wei Qiu
}

\begin{abstract}
Based on the extended Mie theory with Fourier transform, the electromagnetic field in homogeneous gyrotropic media, for the first time, can be analytically obtained in spectral domain in terms of spherical eigne-vectors with their associated coefficients. The coefficients of electromagnetic fields in the gyrotropic shell and the isotropic host medium are thus exactly solved in a recursive manner. Our analytical extended Mie scattering theory have been numerically validated. Using this analytical approach, results of the scattering by the general gyrotropic coated conducting sphere are obtained.
\end{abstract}

Index Terms-Coated structures, electromagnetic scattering, gyrotropic materials.

\section{INTRODUCTION}

One century ago, the electromagnetic scattering theory by a homogeneous isotropic sphere illuminated by a plane wave has been established by Lorenz and Mie [1], [2], respectively. Since then, many scholars extended and developed the Mie theory [3]-[6]. Recently, the scattering characteristics of anisotropic media have aroused increasing interest owing to their wide applications in optical signal processing, radar cross section controlling, radar absorber synthesis, and microwave device fabrication. Much work has been done on the interaction between a plane wave and an anisotropic medium [7]-[16]. Many numerical and analytical methods have been developed to study this problem. For instance, the finite-difference time-domain (FDTD) method [7], [8], the moment method [9], the integral equation method [10], mode expansion method [11], [12], and hybrid finite element-boundary integral-multilevel fast multipole algorithm (FE-BI-MLFMA)[13]. Subsequently, the spherical vector wave functions (SVWFs) expansions along with the Fourier transform were employed to describe the plane wave scattering by a uniaxial anisotropic sphere and a plasma anisotropic sphere [14], [15] based on the plane wave expansion in terms of SVWFs in isotropic medium [17].

However, analytical scattering theory for an optically gyrotropiccoated conducting sphere or even just a single gyrotropic sphere, in which both permittivity and permeability are general gyrotropic tensors, has not been successfully reported. In this connection, this communication proposes an extended Mie theory based on Fourier transform, and tackles this existing problem. Electromagnetic fields in a gyrotropic anisotropic medium can be expanded in terms of SVWFs in gyrotropic anisotropic medium. Applying the boundary conditions at

Manuscript received October 08, 2010; revised February 27, 2011; accepted April 04, 2011. Date of publication August 12, 2011; date of current version November 02, 2011. This work was supported in part by Grant No. 60971047 of the National Natural Science Foundation of China (NSFC) and in part by Grant No. Y1080730 of the Natural Science Foundation of Zhejiang Province of China.

Y. L. Geng is with the Institute of Antenna and Microwaves, Hangzhou Dianzi University, Xiasha, Hangzhou, Zhejiang 310018, China.

C. W. Qiu is with Department of Electrical and Computer Engineering, National University of Singapore, Singapore 117576, Singapore (e-mail: eleqc@nus.edu.sg).

Color versions of one or more of the figures in this communication are available online at http://ieeexplore.ieee.org.

Digital Object Identifier 10.1109/TAP.2011.2164195

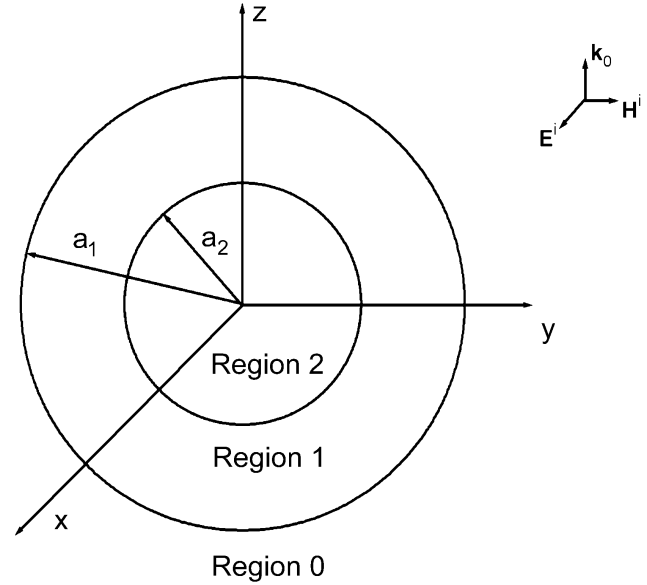

Fig. 1. The geometry of a generalized gyrotropic-coated conducting sphere.

the interface between the free space and the gyrotropic shell and at another interface between the gyrotropic shell and the PEC core, those unknown coefficients associated with eigenvectors in the gyrotropic shell as well as those in free space can be obtained analytically. Then, radar cross sections of a gyrotropic-coated conducting sphere can be readily derived. The theoretical analysis and numerical results show that the present method can be reduced to these of a homogeneous gyrotropic sphere when the radius of the conducting sphere approaches to zero [16]. This analytical solution to electromagnetic scattering by a gyrotropic-coated conducting sphere can be used to characterize the scattering of objects in microwaves as well as in optics, and also be helpful to understand wireless communication channels and radio wave propagation mechanisms.

\section{Formulation OF THE PROBLEM}

Consider a geometry in Fig. 1 which shows a cross section of a gyrotropic-coated conducting sphere (the outer and inner radii of the gyrotropic shell are $a_{1}$ and $a_{2}$, respectively). Three distinct regions are thus divided, namely, region 0 for the free space, region 1 for the gyrotropic spherical shell, and region 2 for the conducting sphere. This composite structure is illuminated by a plane electromagnetic wave (which is assumed to have an electric-field amplitude equal to unity along the $x$-direction, propagating along the $z$-direction). In the following analysis, the time dependence of $\exp (-i \omega t)$ is assumed but suppressed throughout the treatment.

The permittivity and permeability of the generalized gyrotropic material in the shell are characterized by

$$
\overline{\boldsymbol{\epsilon}}=\left[\begin{array}{ccc}
\epsilon_{1} & -i \epsilon_{2} & 0 \\
i \epsilon_{2} & \epsilon_{1} & 0 \\
0 & 0 & \epsilon_{3}
\end{array}\right] ; \quad \overline{\boldsymbol{\mu}}=\left[\begin{array}{ccc}
\mu_{1} & -i \mu_{2} & 0 \\
i \mu_{2} & \mu_{1} & 0 \\
0 & 0 & \mu_{3}
\end{array}\right]
$$

The wave equation in such a source-free and unbounded gyrotropic anisotropic medium can be written as

$$
\nabla \times\left[\overline{\boldsymbol{\mu}}^{-1} \cdot \nabla \times \boldsymbol{E}\right]-\omega^{2} \overline{\boldsymbol{\epsilon}} \cdot \boldsymbol{E}=0
$$

where $\boldsymbol{E}$ denotes the electric field.

Using Fourier transform and the expansion of plane wave in terms of SVWFs in isotropic media [17], one can obtain the electromagnetic 
fields (by the subscript 1$)$ in the gyrotropic shell $\left(a_{2} \leq r \leq a_{1}\right)$ on the basis of the [16]:

$$
\begin{gathered}
\boldsymbol{E}_{1}=\sum_{l=1}^{2} \sum_{q=1}^{2} \sum_{m n n^{\prime}} F_{m n^{\prime} q}^{(l)} \\
\times \int_{0}^{\pi}\left[A_{m n q}^{e}\left(\theta_{k}\right) \boldsymbol{M}_{m n}^{(l)}\left(\boldsymbol{r}, k_{q}\right)+B_{m n q}^{e}\left(\theta_{k}\right) \boldsymbol{N}_{m n}^{(l)}\left(\boldsymbol{r}, k_{q}\right)\right. \\
\left.\quad+C_{m n q}^{e}\left(\theta_{k}\right) \boldsymbol{L}_{m n}^{(l)}\left(\boldsymbol{r}, k_{q}\right)\right] \\
\quad \times P_{n^{\prime}}^{m}\left(\cos \theta_{k}\right) k_{q}^{2} \sin \theta_{k} d \theta_{k} \\
\boldsymbol{H}_{1}=\sum_{l=1}^{2} \sum_{q=1}^{2} \sum_{m n n^{\prime}} F_{m n^{\prime} q}^{(l)} \\
\times \int_{0}^{\pi}\left[A_{m n q}^{h}\left(\theta_{k}\right) \boldsymbol{M}_{m n}^{(l)}\left(\boldsymbol{r}, k_{q}\right)+B_{m n q}^{h}\left(\theta_{k}\right) \boldsymbol{N}_{m n}^{(l)}\left(\boldsymbol{r}, k_{q}\right)\right. \\
\left.\quad+C_{m n q}^{h}\left(\theta_{k}\right) \boldsymbol{L}_{m n}^{(l)}\left(\boldsymbol{r}, k_{q}\right)\right] \\
\quad \times P_{n^{\prime}}^{m}\left(\cos \theta_{k}\right) k_{q}^{2} \sin \theta_{k} d \theta_{k}
\end{gathered}
$$

where $n^{\prime}$ and $n$ are summed up both from 0 to $+\infty$ while $m$ is summed up from $-n$ to $n ; \boldsymbol{r}$ denotes a position vector in the spherical coordinates; the angle $\theta_{k}=\tan ^{-1}\left[\sqrt{\left(k_{x}^{2}+k_{y}^{2}\right)} / k_{z}\right]$; the unknown coefficients, $F_{m n^{\prime} q}^{(l)}$, are to be determined using the boundary conditions; and finally $A_{m n q}^{p}\left(\theta_{k}\right), B_{m n q}^{p}\left(\theta_{k}\right), C_{m n q}^{p}\left(\theta_{k}\right)$ (where $p=e$ or $h$ ) and $k_{q}$ are all functions of $\theta_{k}$ and they have been derived in [16] as shown in the Appendix. The vectors, $\boldsymbol{M}_{m n}^{(l)}, \boldsymbol{N}_{m n}^{(l)}$, and $\boldsymbol{L}_{m n}^{(l)}$, denote SVWFs in isotropic media which are defined as follows [14], [17]

$$
\begin{aligned}
\boldsymbol{M}_{m n}^{(l)}= & z_{n}^{(l)}(k r)\left[i m \frac{P_{n}^{m}(\cos \theta)}{\sin \theta} \widehat{\boldsymbol{\theta}}-\frac{d P_{n}^{m}(\cos \theta)}{d \theta} \widehat{\boldsymbol{\phi}}\right] e^{i m \phi} \\
\boldsymbol{N}_{m n}^{(l)}= & n(n+1) \frac{z_{n}^{(l)}(k r)}{k r} P_{n}^{m}(\cos \theta) e^{i m \phi} \widehat{\boldsymbol{r}}+\frac{1}{k r} \frac{d\left(r z_{n}^{(l)}(k r)\right)}{d r} \\
& \cdot\left[\frac{d P_{n}^{m}(\cos \theta)}{d \theta} \widehat{\boldsymbol{\theta}}+i m \frac{P_{n}^{m}(\cos \theta)}{\sin \theta} \widehat{\boldsymbol{\phi}}\right] e^{i m \phi} \\
\boldsymbol{L}_{m n}^{(l)}= & \left\{\frac{d z_{n}^{(l)}(k r)}{d(k r)} P_{n}^{m}(\cos \theta) \widehat{\boldsymbol{r}}+\frac{z_{n}^{(l)}(k r)}{k r}\right. \\
& \left.\times\left[\frac{d P_{n}^{m}(\cos \theta)}{d \theta} \widehat{\boldsymbol{\theta}}+i m \frac{P_{n}^{m}(\cos \theta)}{\sin \theta} \widehat{\boldsymbol{\phi}}\right]\right\} e^{i m \phi}
\end{aligned}
$$

where $z_{n}^{(l)}(x)$ (with $l=1,2,3$ and 4) denotes an appropriate kind of spherical Bessel functions, $j_{n}(x), y_{n}(x), h_{n}^{(1)}(x)$ and $h_{n}^{(2)}(x)$, respectively.

To characterize the scattering fields of the gyrotropic-coated conducting sphere, the incidence wave (designated by the superscript inc) and the scattered wave (designated by the superscript $s$ ) can be expressed in free space in terms of SVWFs as follows [17]

$$
\begin{aligned}
\boldsymbol{E}^{i n c}= & \sum_{m n}\left[\delta_{m, 1}+\delta_{m,-1}\right] \\
& \times\left[a_{m n}^{x} \boldsymbol{M}_{m n}^{(1)}\left(\boldsymbol{r}, k_{0}\right)+b_{m n}^{x} \boldsymbol{N}_{m n}^{(1)}\left(\boldsymbol{r}, k_{0}\right)\right] \\
\boldsymbol{H}^{i n c}= & \frac{k_{0}}{i \omega \mu_{0}} \sum_{m n}\left[\delta_{m, 1}+\delta_{m,-1}\right] \\
& \times\left[a_{m n}^{x} \boldsymbol{N}_{m n}^{(1)}\left(\boldsymbol{r}, k_{0}\right)+b_{m n}^{x} \boldsymbol{M}_{m n}^{(1)}\left(\boldsymbol{r}, k_{0}\right)\right]
\end{aligned}
$$

and

$$
\begin{aligned}
\boldsymbol{E}^{s} & =\sum_{m n}\left[A_{m n}^{s} \boldsymbol{M}_{m n}^{(3)}\left(\boldsymbol{r}, k_{0}\right)+B_{m n}^{s} \boldsymbol{N}_{m n}^{(3)}\left(\boldsymbol{r}, k_{0}\right)\right] \\
\boldsymbol{H}^{s} & =\frac{k_{0}}{i \omega \mu_{0}} \sum_{m n}\left[A_{m n}^{s} \boldsymbol{N}_{m n}^{(3)}\left(\boldsymbol{r}, k_{0}\right)+B_{m n}^{s} \boldsymbol{M}_{m n}^{(3)}\left(\boldsymbol{r}, k_{0}\right)\right]
\end{aligned}
$$

where $k_{0}$ denotes the wave number in free space, and the expansion coefficients of the incident wave $a_{m n}^{x}, b_{m n}^{x}$, and $\delta_{m n}$ have been obtained earlier in [15] and are defined by

$$
\begin{aligned}
a_{m n}^{x} & = \begin{cases}i^{n+1} \frac{2 n+1}{2 n(n+1)}, & m=1 \\
i^{n+1} \frac{2 n+1}{2}, & m=-1\end{cases} \\
b_{m n}^{x} & = \begin{cases}i^{n+1} \frac{2 n+1}{2 n(n+1)}, & m=1 \\
-i^{n+1} \frac{2 n+1}{2}, & m=-1\end{cases} \\
\delta_{s, l} & = \begin{cases}1, & s=l \\
0, & s \neq l .\end{cases}
\end{aligned}
$$

The expansion coefficients of scattered fields, $A_{m n}^{s}$ and $B_{m n}^{s}$ in (6) ( $n$ varies from 0 to $+\infty$ while $m$ changes from $-n$ to $n$ ), are unknowns to be determined using the boundary conditions, together with the unknown coefficients $F_{m n^{\prime} q}^{(l)}$ (where $l=1,2$ and $\left.q=1,2\right)$ in (3).

From the (3a) and ( $3 \mathrm{~b})$, it implies that when the radius $a_{2}$ of the conducting sphere is infinitely small, the electromagnetic fields in the origin is still finite, but the value of the spherical Bessel functions of the second kind becomes infinitely large in the origin. Therefore the expansion coefficients $F_{m n^{\prime} q}^{(2)}$ of SVWFs in (3a) and (3b) will vanish, and in this connection, the present method in this communication can be automatically reduced to a homogeneous gyrotropic anisotropic sphere, which is the same as that of [16].

Applying continuous boundary conditions of tangential field components at the interface between the gyrotropic anisotropic shell and free space (where $r=a_{1}$ ), and utilizing the orthogonality of the tangential SVWFs [15], the following matrix equation is obtained:

$$
\begin{aligned}
{\left[\begin{array}{llll}
T_{11} & T_{12} & T_{13} & T_{14} \\
T_{21} & T_{22} & T_{23} & T_{24} \\
T_{31} & T_{32} & T_{33} & T_{34} \\
T_{41} & T_{42} & T_{43} & T_{44}
\end{array}\right]_{r=a_{1}}\left[\begin{array}{l}
F_{1}^{(1)} \\
F_{2}^{(1)} \\
F_{1}^{(2)} \\
F_{2}^{(2)}
\end{array}\right] } \\
=\left[\begin{array}{cc}
P_{1} & 0 \\
0 & P_{2} \\
0 & P_{3} \\
P_{4} & 0
\end{array}\right]\left[\begin{array}{l}
A \\
B
\end{array}\right]+\left[\begin{array}{l}
C_{1} \\
C_{2} \\
C_{3} \\
C_{4}
\end{array}\right] .
\end{aligned}
$$

Similarly on the surface of the conducting sphere (where $r=a_{2}$ ), the boundary condition requires the vanishing of the tangential components of the electric field. Hence, we have

$$
\left[\begin{array}{llll}
T_{11} & T_{12} & T_{13} & T_{14} \\
T_{21} & T_{22} & T_{23} & T_{24}
\end{array}\right]_{r=a_{2}} \cdot\left[\begin{array}{l}
F_{1}^{(1)} \\
F_{2}^{(1)} \\
F_{1}^{(2)} \\
F_{2}^{(2)}
\end{array}\right]=0
$$

In the right of (9), 0 can be regarded as a $N \times 1$ matrix, which is

$$
0=\underbrace{[0,0, \cdots, 0]^{t}}_{N}
$$

where $t$ denotes the transpose. In (8) and (9), $T_{l_{q}}$ is $N \times N$ matrix; $F_{q}^{(l)}(l=1,2$ and $q=1,2) ; C_{i}$ and $P_{i}(i=1,2,3,4)$ are $N \times 1$ 
matrix; $A$ and $B$ are $1 \times N$ matrix; and $\mathrm{N}$ is the maximum value of $n$ and $n^{\prime}$ in (3), (5) and (6). They appear to be as the following

$$
\begin{aligned}
& T_{1 q}\left(n, n^{\prime}\right)=\int_{0}^{\pi} A_{m n q}^{e} j_{n}\left(k_{q} r\right) P_{n^{\prime}}^{m}\left(\cos \theta_{k}\right) \\
& \times k_{q}^{2} \sin \theta_{k} d \theta_{k}, \\
& T_{2 q}\left(n, n^{\prime}\right)=\int_{0}^{\pi}\left[B_{m n q}^{e} R_{n}^{(1)}\left(k_{q} r\right)+C_{m n q}^{e} \frac{j_{n}\left(k_{q} r\right)}{r}\right] \\
& \times P_{n^{\prime}}^{m}\left(\cos \theta_{k}\right) k_{q}^{2} \sin \theta_{k} d \theta_{k}, \\
& T_{3 q}\left(n, n^{\prime}\right)=\int_{0}^{\pi} A_{m n q}^{h} j_{n}\left(k_{q} r\right) P_{n^{\prime}}^{m}\left(\cos \theta_{k}\right) \\
& \times k_{q}^{2} \sin \theta_{k} d \theta_{k} \\
& T_{4 q}\left(n, n^{\prime}\right)=\int_{0}^{\pi}\left[B_{m n q}^{h} R_{n}^{(1)}\left(k_{q} r\right)+C_{m n q}^{h} \frac{j_{n}\left(k_{q} r\right)}{r}\right] \\
& \times P_{n^{\prime}}^{m}\left(\cos \theta_{k}\right) k_{q}^{2} \sin \theta_{k} d \theta_{k}, \\
& T_{1,2+q}\left(n, n^{\prime}\right)=\int_{0}^{\pi} A_{m n q}^{e} y_{n}\left(k_{q} r\right) P_{n^{\prime}}^{m}\left(\cos \theta_{k}\right) \\
& \times k_{q}^{2} \sin \theta_{k} d \theta_{k} \\
& T_{2,2+q}\left(n, n^{\prime}\right)=\int_{0}^{\pi}\left[B_{m n q}^{e} R_{n}^{(2)}\left(k_{q} r\right)+C_{m n q}^{e} \frac{y_{n}\left(k_{q} r\right)}{r}\right] \\
& \times P_{n^{\prime}}^{m}\left(\cos \theta_{k}\right) k_{q}^{2} \sin \theta_{k} d \theta_{k}, \\
& T_{3,2+q}\left(n, n^{\prime}\right)=\int_{0}^{\pi} A_{m n q}^{h} y_{n}\left(k_{q} r\right) P_{n^{\prime}}^{m}\left(\cos \theta_{k}\right) \\
& \times k_{q}^{2} \sin \theta_{k} d \theta_{k}, \\
& T_{4,2+q}\left(n, n^{\prime}\right)=\int_{0}^{\pi}\left[B_{m n q}^{h} R_{n}^{(2)}\left(k_{q} r\right)+C_{m n q}^{h} \frac{y_{n}\left(k_{q} r\right)}{r}\right] \\
& \times P_{n^{\prime}}^{m}\left(\cos \theta_{k}\right) k_{q}^{2} \sin \theta_{k} d \theta_{k} \\
& F_{q}^{(l)}=\left[F_{m 1 q}^{(l)}, F_{m 2 q}^{(l)}, \cdots, F_{m N q}^{(l)}\right]^{t} \\
& P_{1}=\left[h_{1}^{(1)}\left(k_{0} a_{1}\right), h_{2}^{(1)}\left(k_{0} a_{1}\right), \cdots, h_{N}^{(1)}\left(k_{0} a_{1}\right)\right]^{t} \\
& P_{2}=\left[R_{1}^{(3)}\left(k_{0} a_{1}\right), R_{2}^{(3)}\left(k_{0} a_{1}\right), \cdots, R_{N}^{(3)}\left(k_{0} a_{1}\right)\right]^{t} \\
& P_{3}=\frac{k_{0}}{i \omega \mu_{0}}\left[h_{1}^{(1)}\left(k_{0} a_{1}\right), h_{2}^{(1)}\left(k_{0} a_{1}\right), \cdots, h_{N}^{(1)}\left(k_{0} a_{1}\right)\right]^{t} \\
& P_{4}=\frac{k_{0}}{i \omega \mu_{0}}\left[R_{1}^{(3)}\left(k_{0} a_{1}\right), R_{2}^{(3)}\left(k_{0} a_{1}\right), \cdots, R_{N}^{(3)}\left(k_{0} a_{1}\right)\right]^{t} \\
& A=\left[A_{m 1}^{s}, A_{m 2}^{s}, \cdots \cdots, A_{m N}^{s}\right] \\
& B=\left[B_{m 1}^{s}, B_{m 2}^{s}, \cdots \cdots, B_{m N}^{s}\right]
\end{aligned}
$$

and

$$
\begin{aligned}
C_{1}(n) & =\left[\delta_{m, 1}+\delta_{m,-1}\right] a_{m n}^{x} j_{n}\left(k_{0} a_{1}\right) \\
C_{2}(n) & =\left[\delta_{m, 1}+\delta_{m,-1}\right] b_{m n}^{x} R_{n}^{(1)}\left(k_{0} a_{1}\right) \\
C_{3}(n) & =\frac{k_{0}}{i \omega \mu_{0}}\left[\delta_{m, 1}+\delta_{m,-1}\right] b_{m n}^{x} j_{n}\left(k_{0} a_{1}\right) \\
C_{4}(n) & =\frac{k_{0}}{i \omega \mu_{0}}\left[\delta_{m, 1}+\delta_{m,-1}\right] a_{m n}^{x} R_{n}^{(1)}\left(k_{0} a_{1}\right) .
\end{aligned}
$$

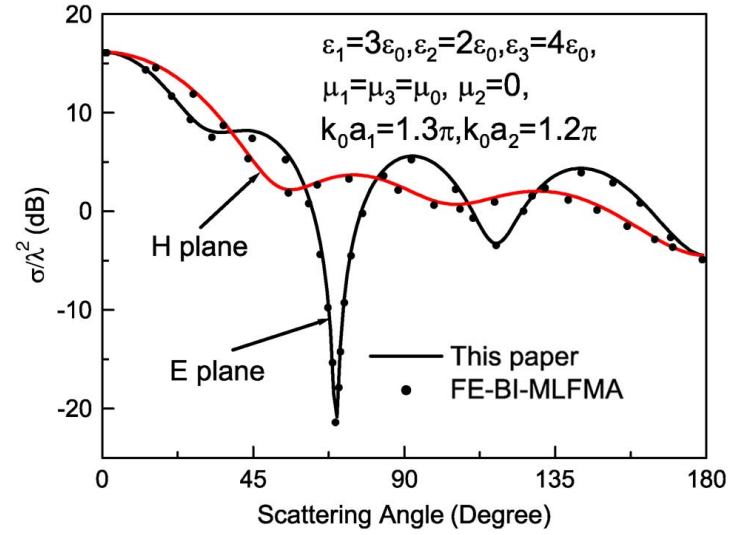

Fig. 2. Radar cross sections (RCSs) versus scattering angle $\theta$ (in degrees) of a non-magnetic case: Results of this communication (solid curve) and FE-BIMLFMA method (circle dot).

In (11)to (15), $m$ is an arbitrary integer, and the radial function $R_{n}^{(l)}(x)$ has the following expression $R_{n}^{(l)}(x)=(1 / x)(d / d x)\left[x z_{n}^{(l)}(x)\right]$. From (8) to (15), it shows that there are six equations and six unknown coefficients, namely, $F_{q}^{(l)}$ (where $q=1,2$ and $l=1,2$ ), $A$ and $B$ in (12)to (14). Thus all unknown coefficients of electromagnetic fields in the gyrotropic anisotropic spherical medium can be obtained. Then the coefficients of scattered fields in free space are calculated and the radar cross section of an anisotropic gyrotropic-coated conducting sphere by a plane wave can be derived.

\section{NUMERICAL RESULTS}

Since there are no reported analytical or numerical results for the scattering of a general gyrotropic-coated conducting sphere, we performed two verifications to validate our analytical method. Firstly, we compared our result for a gyroelectric case with a computational method based on the FE-BI-MLFMA [13] as shown in Fig. 2. In [13], the scattering of the gyroelectric-coated PEC sphere is solved numerically. It is a good candidate to compare with the results from our analytical methods for the more general gyrotropic-coated PEC sphere. Good agreement has been observed.

Secondly, the radius of the conducting sphere is assumed to be extremely small (e.g., $k_{0} a_{2}=0.002 \pi$ ) which tends to a homogeneous gyrotropic sphere [16]. The degenerated results based on the present method agree well with the direction calculation [16] as shown in Fig. 3. Both trials partially verify our theory as well as the Fortran program codes in both $E$ - and $H$-planes.

We also obtained some new results unavailable elsewhere in literature. Two examples are considered herein, and their radar cross sections are plotted in Figs. 4 and 5.

Fig. 4 depicts RCS of a lossless gyrotropic-coated conducting sphere. The electric size of the gyrotropic-coated sphere is chosen as $k_{0} a_{1}=2.1 \pi$ and $k_{0} a_{2}=2 \pi$. The maximum number $n^{\prime}$ in (11) and (15), to ensure a good convergence, is found to be 14 . To illustrate further applicability of the solution to electromagnetic scattering by an electrically large gyrotropic-coated conducting sphere (for example, in its resonance region), the RCS of a lossy gyrotropic-coated sphere of relatively larger size with $k_{0} a_{1}=4 \pi$ and $k_{0} a_{2}=3.9 \pi$, are obtained and depicted in both the $E$-plane and the $H$-plane in Fig. 5. As the electric dimension of the sphere is increased, the maximum number of $n^{\prime}$ used in (11) and (15) must be increased to 18 to achieve the convergence. It is noted that for a lossy gyrotropic-coated conducting sphere of large electrical dimension, the oscillation of RCS in the backward directions will be more suppressed, which can eventually be 


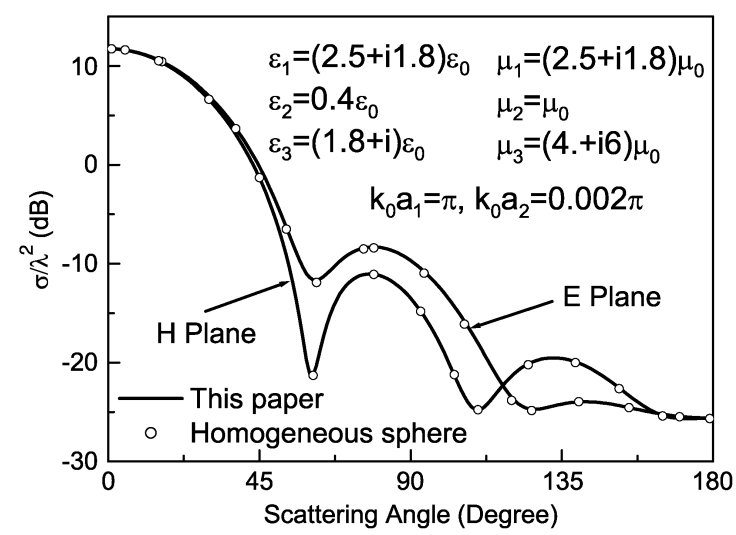

Fig. 3. Radar cross sections (RCSs) versus scattering angle $\theta$ (in degrees): Results of this communication (solid curve) and homogeneous gyrotropic sphere (circle dot).

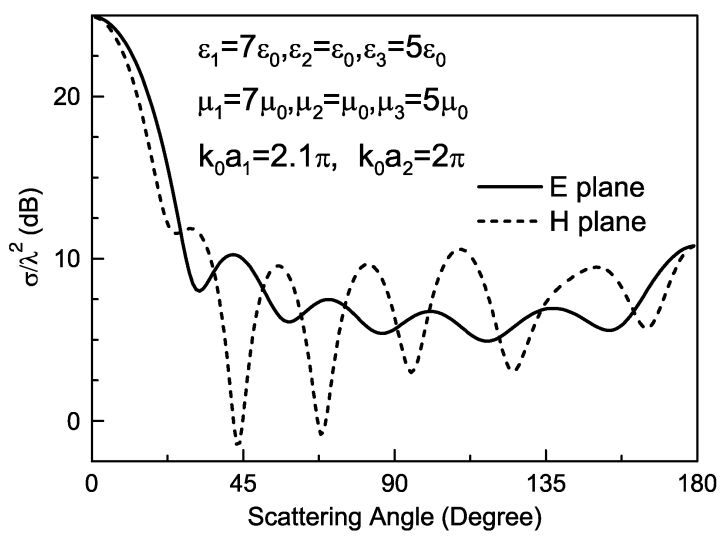

Fig. 4. Radar cross sections (RCSs) versus scattering angle $\theta$ (in degrees) in the $E$-plane (solid curve) and in the $H$-plane (dashed curve) in a lossless case.

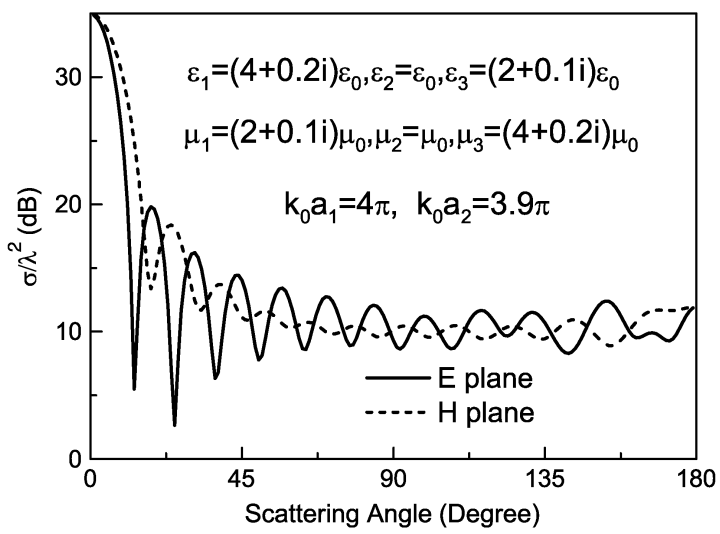

Fig. 5. Radar cross sections (RCSs) versus scattering angle $\theta$ (in degrees) in the $E$-plane (solid curve) and in the $H$-plane (dashed curve) in a lossy case when the size is even larger than that in Fig. 4.

approximated by the geometrical optics limit [11] when the electrical size is further increased.

\section{CONCLUSIONS}

An extended Mie theory based on Fourier transform and SVWFs expansion technique is successfully has been established to model the scattering by a gyrotropic-coated conducting sphere in this communication. The solution has only one-dimensional integral which can be easily evaluated using Gauss integral [18]. The theoretical analysis and numerical results demonstrate the correctness, advantages, and uniqueness since it provides, for the first time, an analytical approach for such a generalized complex media in a coated structure. In addition, the general numerical results, including the lossless and lossy gyrotropiccoated conducting sphere in resonance region, are given. The scattering control by the gyrotropy ratio is under further investigation.

\section{APPENDIX}

The unknown coefficients $A_{m n q}^{e}, B_{m n q}^{e}$ and $C_{m n q}^{e}$ in (3a) can be expressed as $A_{m n q}^{e}=A_{m n q}^{e 1}+A_{m n q}^{e 2}, B_{m n q}^{e}=B_{m n q}^{e 1}+B_{m n q}^{e 2}$, and $C_{m n q}^{e}=C_{m n q}^{e 1}+C_{m n q}^{e 2}$. One can now obtain the expansion coefficients of $\mathbf{E}$-fields in a gyrotropic anisotropic medium as follows: for $p=1$ and $m \geq 0$

$$
\begin{aligned}
A_{m n q}^{e 1}= & i^{n} \frac{2 n+1}{2 n(n+1)} \frac{(n-m) !}{(n+m) !} \frac{\triangle_{1}}{\triangle} \\
& \times\left[(n+m)(n-m+1) P_{n}^{m-1}\left(\cos \theta_{k}\right)\right. \\
& \left.\quad-P_{n}^{m+1}\left(\cos \theta_{k}\right)\right] \\
B_{m n q}^{e 1}= & i^{n} \frac{1}{2 n(n+1)} \frac{(n-m) !}{(n+m) !} \frac{\triangle_{1}}{\triangle} \\
\times & {\left[(n+1)(n+m)(n+m-1) P_{n-1}^{m-1}\left(\cos \theta_{k}\right)\right.} \\
& \quad+(n+1) P_{n-1}^{m+1}\left(\cos \theta_{k}\right) \\
& \quad+n(n-m+2)(n-m+1) P_{n+1}^{m-1}\left(\cos \theta_{k}\right) \\
& \left.\quad+n P_{n+1}^{m+1}\left(\cos \theta_{k}\right)\right] \\
C_{m n q}^{e 1}=i^{n} \frac{1}{2 k_{q}} & \frac{(n-m) ! \triangle_{1}}{(n+m) !} \triangle \\
\times & (n+m)(n+m-1) P_{n-1}^{m-1}\left(\cos \theta_{k}\right) \\
& \quad+P_{n-1}^{m+1}\left(\cos \theta_{k}\right) \\
& \quad-(n-m+2)(n-m+1) P_{n+1}^{m-1}\left(\cos \theta_{k}\right) \\
& \left.\quad-P_{n+1}^{m+1}\left(\cos \theta_{k}\right)\right]
\end{aligned}
$$

while for $p=1$ and $m>0$

$$
\begin{aligned}
& A_{-m n q}^{e 1}=(-1)^{m} \frac{(n+m) !}{(n-m) !} A_{m n q}^{e 1} \\
& B_{-m n q}^{e 1}=(-1)^{m+1} \frac{(n+m) !}{(n-m) !} B_{m n q}^{e 1} \\
& C_{-m n q}^{e 1}=(-1)^{m+1} \frac{(n+m) !}{(n-m) !} C_{m n q}^{e 1} .
\end{aligned}
$$

Similarly, for $p=2$ and $m \geq 0$, we have

$$
\begin{aligned}
A_{m n q}^{e 2}= & i^{n+1} \frac{2 n+1}{n(n+1)} \frac{(n-m) !}{(n+m) !} \\
\times & \begin{aligned}
\frac{\triangle_{2}}{2 \triangle} & {\left[(n+m)(n-m+1) P_{n}^{m-1}\left(\cos \theta_{k}\right)\right.} \\
& \left.\left.\quad+P_{n}^{m+1}\left(\cos \theta_{k}\right)\right]+m P_{n}^{m}\left(\cos \theta_{k}\right)\right\}
\end{aligned}
\end{aligned}
$$




$$
\begin{aligned}
& B_{m n q}^{e 2}=i^{n+1} \frac{1}{n(n+1)} \frac{(n-m) !}{(n+m) !} \\
& \times\left\{\frac { \triangle _ { 2 } } { 2 \triangle } \left[(n+1)(n+m)(n+m-1) P_{n-1}^{m-1}\right.\right. \\
& \times\left(\cos \theta_{k}\right)-(n+1) P_{n-1}^{m+1}\left(\cos \theta_{k}\right) \\
& +n(n-m+2)(n-m+1) \\
& \left.\times P_{n+1}^{m-1}\left(\cos \theta_{k}\right)-n P_{n+1}^{m+1}\left(\cos \theta_{k}\right)\right] \\
& +\left[n(n-m+1) P_{n+1}^{m}\left(\cos \theta_{k}\right)\right. \\
& \left.\left.-(n+1)(n+m) P_{n-1}^{m-1}\left(\cos \theta_{k}\right)\right]\right\} \\
& C_{m n q}^{e 2}=i^{n+1} \frac{1}{k_{q}} \frac{(n-m) !}{(n+m) !} \\
& \times\left\{\frac { \triangle _ { 2 } } { 2 \triangle } \left[(n+m)(n+m-1) P_{n-1}^{m-1}\left(\cos \theta_{k}\right)\right.\right. \\
& -P_{n-1}^{m+1}\left(\cos \theta_{k}\right)-(n-m+2) \\
& \times(n-m+1) P_{n+1}^{m-1}\left(\cos \theta_{k}\right) \\
& \left.+P_{n+1}^{m+1}\left(\cos \theta_{k}\right)\right] \\
& \left.-(2 n+1) \cos \theta_{k} P_{n}^{m}\left(\cos \theta_{k}\right)\right\}
\end{aligned}
$$

while for $p=2$ and $m>0$

$$
\begin{aligned}
& A_{-m n q}^{e 2}=(-1)^{m+1} \frac{(n+m) !}{(n-m) !} A_{m n q}^{e 2} \\
& B_{-m n q}^{e 2}=(-1)^{m} \frac{(n+m) !}{(n-m) !} B_{m n q}^{e 2} \\
& C_{-m n q}^{e 2}=(-1)^{m} \frac{(n+m) !}{(n-m) !} C_{m n q}^{e 2} .
\end{aligned}
$$

Also, one has

$$
\begin{aligned}
\triangle_{1}= & i\left(b_{1} a_{2}+b_{2} a_{1}\right) k_{q}^{2} \sin \theta_{k} \cos \theta_{k} \\
\triangle_{2}= & {\left[b_{1} b_{3} k_{q}^{2} \sin ^{2} \theta_{k}+\left(b_{1}^{2}-b_{2}^{2}\right) k_{q}^{2} \cos ^{2} \theta_{k}\right] k_{q}^{2} \sin \theta_{k} \cos \theta_{k} } \\
& -\left(b_{1} a_{1}+b_{2} a_{2}\right) \\
\triangle= & -\left(b_{2} k_{q}^{2} \cos ^{2} \theta_{k}+a_{2}\right)^{2}+\left(b_{1} k_{q}^{2} \cos ^{2} \theta_{k}-a_{1}\right) \\
& \times\left(b_{1} k_{q}^{2} \cos ^{2} \theta_{k}+b_{3} k_{q}^{2} \sin ^{2} \theta_{k}-a_{1}\right)
\end{aligned}
$$

and

$$
\begin{aligned}
& a_{1}=\omega^{2} \epsilon_{1} ; a_{2}=\omega^{2} \epsilon_{2} ; a_{3}=\omega^{2} \epsilon_{3} \\
& b_{1}=\frac{\mu_{1}}{\mu_{1}^{2}-\mu_{2}^{2}} ; b_{2}=\frac{\mu_{2}}{\mu_{1}^{2}-\mu_{2}^{2}} ; b_{3}=1 / \mu_{3} .
\end{aligned}
$$

Similarly, the coefficients $A_{m n q}^{h}, B_{m n q}^{h}$ and $C_{m n q}^{h}$ in (3b) can also be obtained for the gyrotropic anisotropic medium.

\section{ACKNOWLEDGMENT}

The authors are indebted to Prof. X. Q. Sheng and Dr. Z. Peng from the Beijing Institute of Technology for sending them their data.

\section{REFERENCES}

[1] L. Lorenz, "Sur la Lumière réfléchie et réfractée par une sphère transparente," Vidensk. Selsk. Skrifter, vol. 6, pp. 1-62, 1890.

[2] G. Mie, "Beitrage zur Optik truber Medien speziell kolloidaler Metallosungen," Ann. Phys., vol. 25, pp. 377-455, 1908.

[3] A. L. Aden and M. Kerker, "Scattering of electromagnetic wave from two concentric spheres," J. Appl. Phys., vol. 22, pp. 1242-1246, 1951.

[4] J. H. Richmond, "Scattering by a ferrite-coated conducting sphere," IEEE Trans. Antennas Propag., vol. 35, no. 1, pp. 73-79, 1987.

[5] J. D. Keener, K. J. Chalut, J. W. Pyhtila, and A. Wax, "Application of Mie theory to determine the structure of spheroidal scatterers in biological materials," Opt. Lett., vol. 32, no. 10, pp. 1326-1328, 2007.

[6] B. Garcia-Camara, F. Moreno, F. Gonzalez, J. M. Saiz, and G. Videen, "Light scattering resonances in small particles with electric and magnetic properties," J. Opt. Soc. Am. A, vol. 25, no. 2, pp. 327-334, 2008.

[7] A. Taflove, "Review of the formulation and applications of the finite-difference-time-domain method for numerical modeling of electromagnetic wave interactions with arbitrary structures," Wave Motion, vol. 10, pp. 547-582, Dec. 1988.

[8] L. A. Dou and A. R. Sebak, "3D FDTD method for arbitrary anisotropic materials," Microwave Opt. Technol. Lett., vol. 48, pp. 2083-2090, 2006.

[9] R. D. Graglia, P. L. E. Uslenghi, and R. S. Zich, "Moment method with isoparametric elements for three-dimensional anisotropic scatterers," Proc. IEEE, vol. 77, no. 5, pp. 750-760, May 1989.

[10] S. N. Papadakis, N. K. Uzunoglu, and C. N. Capsalis, "Scattering of a plane wave by a general anisotropic dielectric ellipsoid," J. Opt. Soc. Am. A, vol. 7, pp. 991-997, 1990.

[11] C.-W. Qiu, S. Zouhdi, and A. Razek, "Modified spherical wave functions with anisotropy ratio: Application to the analysis of scattering by multilayered anisotropic shells," IEEE Trans. Antennas Propag., vol. 55, no. 12, pp. 3515-3523, 2007.

[12] Z. Lin and S. T. Chui, "Electromagnetic scattering by optically anisotropic magnetic particle," Phys. Rev. E, vol. 69, p. 056614, 2004.

[13] X. Q. Sheng and Z. Peng, "Analysis of scattering by large objects with off-diagonally anisotropic material using finite element-boundary integral-multilevel fast multipole algorithm," IET Microw. Antennas Propag., vol. 4, pp. 492-500, 2010.

[14] Y. L. Geng, X. B. Wu, L. W. Li, and B. R. Guan, "Mie scattering by a uniaxial anisotropic sphere," Phys. Rev. E, vol. 70, no. 5, p. 056609, 2004.

[15] Y. L. Geng, X. B. Wu, and L. W. Li, "Analysis of electromagnetic scattering by a plasma anisotropic sphere," Radio Sci., vol. 38, no. 6, pp. 1104/1-1104/12, 2003.

[16] Y. L. Geng and C. W. Qiu, Analytical Spectral-Domain Scattering Theory of a General Gyrotropic Sphere [Online]. Available: http://www.arxiv.com/abs/1102.4057 2011

[17] D. Sarkar and N. J. Halas, "General vector basis function solution of Maxwell's equations," Phys. Rev. E, vol. 56, pp. 1102-1112, 1997.

[18] M. Abramowitz and I. A. Stegun, Handbook of Mathematical Functions with Formulas, Graphs, and Mathematical Tables. New York: Dover Publications, 1972. 